\title{
DETERMINATION OF BLOOD CELLS MOTIONS AND INTERACTIONS BY A CONFOCAL MICRO-PTV SYSTEM
}

\author{
Rui LIMA (1), Takuji ISHIKAWA (2), Yohsuke IMAI (2), Motohiro TAKEDA (2, 3), \\ Shigeo WADA (4), Takami YAMAGUCHI (2)
}

\begin{abstract}
1. Dep. Mechanical Technology, ESTiG, Bragança Polytechnic, C. Sta. Apolonia, 5301-857 Bragança, Portugal; 2. Depart. of Bioengineering and Robotics, Grad. Sch. Eng., Tohoku University, 6-6-01 Aoba, 980-8579 Sendai, Japan; 3. Div. of Surgical Oncology, Grad. Sch. Medicine, Tohoku University, 2-1 Seiryo-machi, Aoba-ku, 9808575 Sendai, Japan; 4. Dep. of Mechanical Science and Bioengineering, Grad. Sch. of Eng. Science., Osaka University, Toyonaka, 560-8531 Osaka, Japan.
\end{abstract}

\section{Introduction}

The development of optical experimental techniques has contributed to obtain explanations on the way the blood flows through microvessels. [Goldsmith \& Turitto 1986, Lima 2007]. Although the past results have been encouraging, detailed studies on the flow properties of blood in the microcirculation has been limited by several technical factors such as poor spatial resolution and difficulty to obtain quantitative detailed measurements at such small scales. In recent years, due to advances in computers, confocal microscopy, and digital image processing techniques, it has become possible to combine a micro-particle tracking velocimetry (PTV) system with a confocal microscope. The present study shows for the first time confocal micro-PTV measurements of the dynamic flow behaviour of red blood cells (RBCs) in concentrated suspensions. The measurements were performed at several depths of a $100 \mu \mathrm{m}$ glass capillaries

\section{Materials and Methods}

Working fluids: In this study we used dextran 40 (Dx40) containing Hcts from 2 to $35 \%$ of RBCs and about $2 \%$ of white blood cells (WBCs). The blood cells were labeled with a lipophilic carbocyanime derivative (CM-Dil, C-7000, Molecular Probes) [Lima 2007].

Experimental set-up: The confocal micro-PTV system consists of an inverted microscope (IX71, Olympus) combined with a confocal scanning unit (CSU22, Yokogawa), a diode-pumped solid state (DPSS) laser (Laser Quantum Ltd) with an excitation wavelength of $532 \mathrm{~nm}$ and a high-speed camera (Phantom v7.1) [Lima et al. 2006, Lima et al. 2007]. The microchannel was placed on the stage of the microscope, with a controlled temperature of about $37^{\circ} \mathrm{C}$, where the flow rate was kept constant by using a syringe pump. The confocal images were captured at a rate of 100 frames/s and then evaluated in Image J (NIH) by using a manual tracking MTrackJ plugin [Lima 2007].

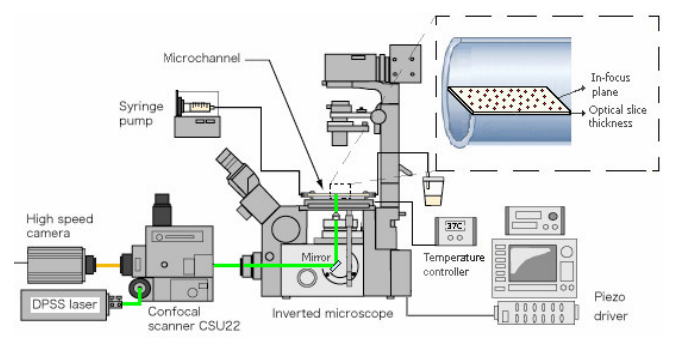

Figure 1: Confocal micro-PTV experimental set-up.

\section{Results and Discussion}

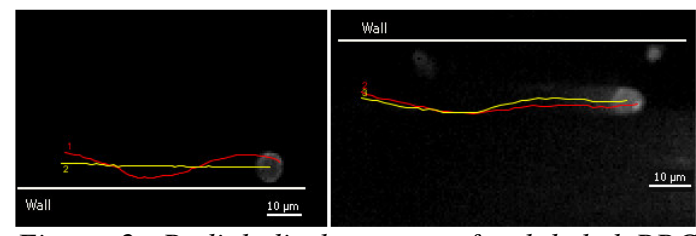

Figure 2: Radial displacement of a labeled RBC with both translational and rotational motion: with (right side) and without interaction (left side).

Figure 2 shows the orientation of two RBCs with and without interaction measured around plasma layer of concentrated suspensions. By using our confocal system, it was possible to measure both translational and rotational motion; translational motion was measured around RBC centre, whereas rotation was measured along the membrane. Our results demonstrate the ability of the confocal micro-PTV system to obtain both translational and rotational motion of individual RBCs flowing in concentrated suspensions. Owing to its optical sectioning ability and consequent improvement of the image contrast and definition, the proposed confocal system can provide additional detailed description on the RBC motion not obtainable by other conventional methods.

\section{References}

Goldsmith \& Turitto, Thromb Haemost, 55, 415435, 1986.

Lima et al, Mea. Sci Tech, 17: 797- 808, 2006.

Lima et al, J. Biomech., 40: 2752-2757, 2007.

Lima R., PhD Thesis, Tohoku Univ, 2007. 